\title{
Enhanced oxidative stress in hypertrophic cardiomyopathy
}

\author{
Paweł P. Dimitrow ${ }^{1}$, Anetta Undas ${ }^{2}$, Paweł Wołkow ${ }^{3}$, Wiesława Tracz ${ }^{2}$, \\ Jacek S. Dubiel ${ }^{1}$ \\ ${ }^{1}$ 2nd Department of Cardiology, Collegium Medicum, Jagiellonian University, Kopernika 17, PL 31-501 Kraków, \\ Poland \\ ${ }^{2}$ Department of Cardiac and Vascular Disease, Collegium Medicum Jagiellonian University, Prądnicka 80, \\ PL 31-202 Kraków, Poland \\ ${ }^{3}$ Department of Pharmacology, Collegium Medicum, Jagiellonian University, Grzegórzecka 16, PL 31-531 Kraków \\ Poland
}

Correspondence: Paweł P. Dimitrow, e-mail: dimitrow@mp.pl

\begin{abstract}
:
Elevated plasma levels of inflammation and endothelial dysfunction markers have been reported in patients with hypertrophic cardiomyopathy (HCM). The aim of the current study was to determine whether HCM is associated with enhanced oxidative stress. We enrolled 54 HCM patients with sinus rhythm, including 21 subjects with a left ventricular outflow tract (LVOT) obstruction (gradient $\geq 30 \mathrm{mmHg}$ ), and 54 age- and sex-matched controls without cardiovascular diseases. Serum levels of 8-isoprostaglandin $\mathrm{F}_{2 \alpha}$ (8-iso-PGF $2 \alpha$ ), a stable marker of oxidative stress, were determined.

Serum 8-iso-PGF $2 \alpha$ levels were elevated in $\mathrm{HCM}$ patients compared with controls $(35.4 \pm 10.2$ vs. $29.9 \pm 9.9 \mathrm{pg} / \mathrm{ml}, \mathrm{p}<0.001)$. Patients with obstructive HCM displayed higher 8-iso- $\mathrm{PGF}_{2 \alpha}$ levels compared with the non-obstructive HCM subgroup (41.6 \pm 12.7 vs. $31.4 \pm 5.4 \mathrm{pg} / \mathrm{ml}, \mathrm{p}<0.0001$ ). Both anatomic (mitral-septal distance) and hemodynamic (subaortic gradient) indexes of LVOT obstruction, but not other echocardiographic variables, correlated with 8 -iso- $\mathrm{PGF}_{2 \alpha}$ levels $(\mathrm{r}=-0.43 ; \mathrm{p}<0.05$ and $\mathrm{r}=0.39 ; \mathrm{p}<0.05$, respectively).

This study is the first to show that HCM is characterized by enhanced oxidative stress as evidenced by higher 8 -iso-PGF $2 \alpha$, which achieves its highest values in the presence of LVOT obstruction in HCM patients.
\end{abstract}

Key words:

hypertrophic cardiomyopathy, oxidative stress

Abbreviations: HCM - hypertrophic cardiomyopathy, LV - left ventricular, LVOT - left ventricular outflow tract

\section{Introduction}

8-Isoprostaglandin $\mathrm{F}_{2 \alpha}$ (8-iso- $\left.\mathrm{PGF}_{2 \alpha}\right)$ is produced upon nonenzymatic arachidonic acid peroxidation. Plasma levels of this reliable marker of oxidative stress are elevated in patients with coronary artery disease [18] and those with acute myocardial infarction [4]. Similarly, oxidative stress is enhanced in diabetes, inflammatory disorders, infections, renal failure and heart failure $[5,15]$. Reactive oxygen species cause hypertrophy, injury, apoptosis and necrosis in cardiac myocytes $[11,14]$. Interestingly, oxidative stress has been reported to be increased in experimental models of cardiac hypertrophy induced by left ventricular pressure overload [6, 7, 14]. 
To our knowledge, there is only a single report on oxidative stress determined in HCM patients [13]. Nakamura et al. [13] studied HCM patients with left ventricular (LV) systolic dysfunction and documented increased expression of 4-hydroxy-2-nonenal-modified protein, a marker of oxidative stress in the myocardium. However, HCM patients with LV systolic dysfunction occur infrequently in the general HCM population (5-10\%), and myocardial biopsies are rarely performed in countries other than Japan. Blood markers of oxidative stress were not determined in the study by Nakamura et al. [13].

It is not clear whether HCM might be associated with enhanced oxidative stress when measured in circulating blood. Thus, we sought to assess 8-iso$\mathrm{PGF}_{2 \alpha}$ as a reliable marker of oxidative stress in patients with the most common HCM features, i.e. nondilated LV with hypertrophied wall and normal LV systolic function. An additional aim of the study was to determine the effect of LVOT obstruction on oxidative stress.

\section{Materials and Methods}

\section{Study population}

We enrolled 54 HCM patients (31 males and 23 females, mean age $47 \pm 15$ years) with sinus rhythm. The diagnosis of HCM was based on typical clinical, electrocardiographic and echocardiographic features. All HCM patients had normal LV systolic function and nondilated LV cavity. Patients were treated with verapamil $(n=19)$ or beta-blockers $(n=21)$, and 14 patients who were referred for the initial evaluation received no medication. The control group included 54 subjects matched for age and sex (31 males and 23 females, mean age $45 \pm 13$ years) who were recruited from the hospital staff. Exclusion criteria were acute illness, arterial hypertension, cancer, paroxysmal or persistent atrial fibrillation, endocarditis, previous cerebrovascular event, myocardial infarction, renal or hepatic dysfunction, and diabetes mellitus.

Coronary angiography was performed in 12 patients and normal coronary arteries were detected. As in the study by Thaman et al. [17], only patients with typical chest pain or risk factors for coronary artery disease underwent coronary angiography. Conse- quently, not all patients, but only selected subjects, underwent coronary angiography, which yielded normal results. Therefore, it is unlikely that coronary artery disease was an important confounding factor in the analyses performed in patients with $\mathrm{HCM}$ or in the control group (subjects without any cardiac symptoms).

\section{Echocardiographic study}

Transthoracic echocardiographic examinations were performed using a Vivid 7 ultrasound machine (GEMedical Systems, Inc., Milwaukee, WI, USA).

In each patient, M-mode and two-dimensional echocardiograms were obtained, followed by pulsed and continuous-wave Doppler recordings. Conventional techniques were used to measure the LV size. The LV contractility was assessed by fractional shortening as recommended previously [17]. The LVOT gradient was measured using Doppler recordings, and a value of $>30 \mathrm{mmHg}$ was considered a clinically significant cut-off value. Particular care was taken to report only the gradients derived from Doppler velocity profiles typical of subaortic obstruction, thus avoiding contamination with the jet of mitral regurgitation. Morphological assessment of the narrowed LVOT was performed by measurement of the minimal distance between the mitral valve and ventricular septum during systole in the parasternal long-axis view with M-mode echocardiography [9].

A LVOT gradient $\geq 30 \mathrm{mmHg}$ was detected in 21 patients.

Echocardiographic evaluation in the control group revealed no abnormalities of cardiac structure and function. The septal thickness was $\leq 11 \mathrm{~mm}$ in all control subjects.

All patients and control subjects gave their informed consent. The Jagiellonian University Ethical Committee approved the study.

Blood was taken immediately after echocardiographic examination. Blood samples were taken with minimal stasis between 8 and $9 \mathrm{AM}$ after an overnight fast. Serum samples were centrifuged at $2,000 \times \mathrm{g}$ for 20 minutes and stored in aliquots at $-80^{\circ} \mathrm{C}$ until analysis. Serum levels of 8 -iso-PGF $2 \alpha$, a marker of oxidative stress, were determined using an immunoenzymatic test (Cayman Chemicals, Ann Arbor, MI) according to the manufacturer's instructions. The intra-assay and interassay coefficients of variation were $3.5 \%$ and $3.9 \%$, respectively. 
Statistical analysis. Data were given as the mean \pm SD. The Kolmogorov-Smirnov test was used to assess conformity with a normal distribution. The Student's $t$-test was used to test differences between groups. The Pearson correlation coefficient was calculated to identify significant associations between variables. Multivariable linear regression analyses were performed to identify variables significantly associated with 8-iso-PGF $\mathrm{PG}_{2 \alpha}$. Independent variables that remained significantly associated with 8 -iso- $\mathrm{PGF}_{2 \alpha}$ after a stepwise selection procedure were retained in the final models. Plots of studentized residuals versus predicted values were inspected to evaluate fit of the model and potential violations of the model assumptions. A p-value $<0.05$ was considered statistically significant.

\section{Results}

The HCM patients and controls did not differ with regard to the number of cigarette smokers (by 8 subjects in each group). There was no difference in age between the HCM patients and controls $(47 \pm 15$ vs. $45 \pm 13$ years).

On echocardiography, both LV contractility (fractional shortening $44 \pm 8 \%$ ) and cavity size were normal when analyzed for the entire HCM group. In all HCM patients, the ventricular septum thickness was markedly increased to $24.1 \pm 3.8 \mathrm{~mm}$, whereas the thickness of the posterior wall was within normal range at $11 \pm 2 \mathrm{~mm}$. The septum/posterior wall thickness ratio was $2.3 \pm 0.4$, indicating an asymmetric pattern of LV hypertrophy in all patients. The left atrial diameter was increased by $45 \pm 5 \mathrm{~mm}$.

Serum 8-iso- $\mathrm{PGF}_{2 \alpha}$ levels were elevated in the HCM patients compared with controls $(35.4 \pm 10.2 v s$. $29.9 \pm 9.9 \mathrm{pg} / \mathrm{ml}, \mathrm{p}<0.001)$. Moreover, the obstructive HCM subgroup displayed higher values of 8 -isoprostanes compared with the non-obstructive HCM subgroup $(41.6 \pm 12.7$ vs. $31.4 \pm 5.4 \mathrm{pg} / \mathrm{ml}$; $\mathrm{p}<0.0001$ ). Both anatomic (mitral-septal distance) and hemodynamic (subaortic gradient) indexes of LVOT obstruction correlated with 8-iso- $\mathrm{PGF}_{2 \alpha}$ levels $(\mathrm{r}=-0.43 ; \mathrm{p}<0.05$ and $\mathrm{r}=0.39 ; \mathrm{p}<0.05$, respectively). Other echocardiographic parameters did not correlate with 8 -iso-PGF $2 \alpha$. The level of 8 -iso- $\mathrm{PGF}_{2 \alpha}$ correlated with age $(r=0.44 ; p<0.05)$. Multivariable regression analysis with a stepwise selection procedure in 54 patients with $\mathrm{HCM}$ showed that serum 8 -iso- $\mathrm{PGF}_{2 \alpha}$ concentrations were independently predicted by only age (estimate, $0.21 ; \mathrm{SE}, 0.08 ; \mathrm{p}=0.013$ ) and LVOT gradient (estimate, 0.13; SE, 0.04; $\mathrm{p}=0.005$ ).

\section{Discussion}

Our results showed that HCM is characterized by enhanced oxidative stress and that the LVOT gradient is the independent predictor of serum 8-iso- $\mathrm{PGF}_{2 \alpha}$ levels, used as a marker of oxidative stress. In HCM, pressure overload is present in patients with LVOT obstruction, generating a subvalvular gradient. In previous studies $[6,7,14]$, oxidative stress was elevated in experimental models of LV pressure overload, and the mechanisms of this association have been examined. It has been demonstrated that pressure overload triggers nitric oxide synthase-3 uncoupling as a prominent source of myocardial reactive oxygen species [16]. Antioxidant treatment with tetrahydrobiopterin has prevented nitric oxide synthase- 3 uncoupling and inhibited reactive oxygen species [16]. Moreover [12], tetrahydrobiopterin treatment has been shown to reduce oxidant stress and simultaneously block or reverse maladaptive remodelling in hearts with advanced hypertrophy induced by pressure overload.

There is a relationship between oxidative stress and inflammation in cardiovascular systems under pressure overload. Kai et al. [8] documented that pressure overload induces superoxide production, mainly in the intramyocardial arterial wall. The marker of reactive oxygen species production (4-hydroxy-2-nonenalmodified protein) was also observed in the myocardium with a similar time course. Moreover, Kai et al. [8] have tested candesartan (angiotensin receptor blocker) at a dose that did not reduce blood pressure (antioxidant not hypotensive effect). This subdepressor dose of candesartan reduced the pressure overload reactive oxygen species production associated with the inhibition of monocyte chemoattractant protein-1 induction and macrophage infiltration in early phases of the experiment. In late phases of the experiment, candesartan prevented myocardial fibrosis, but not myocardial hypertrophy. These results suggested that the pressure overload-induced reactive oxygen species production mediated perivascular inflammation 
and the subsequent cardiac fibrosis [8]. The biochemical effect of reactive oxygen species was assessed in other experimental models. Henderson et al. [6] documented that pressure overload leads to increased oxidative stress with redox imbalance, causing increased expression/activity of matrix metalloproteinase-2/9 and a decrease in antioxidant expression producing collagen degradation.

To summarize, mechanical stress by pressure overload and humoral factors (including inflammatory mediators) are linked to the induction of oxidative stress.

In previous studies, we reported that HCM, especially its obstructive form, is associated with reduced endothelium-dependent vasodilatation [3] and with elevated levels of markers of endothelial dysfunction [2] and inflammation [1], which might enhance oxidative stress. It remains to be elucidated whether there is a relationship between markers of oxidative stress, inflammation and endothelial dysfunction in HCM patients with pressure overload due to LVOT obstruction. Further analysis of markers for oxidative stress should include additional markers, e.g. 2,3-dinor-5,6dihydro-15-F(2t)-isoprostane.

In the previous study [13], the level of 4-hydroxy2-nonenal-modified protein (marker of oxidative stress) was significantly increased in HCM patients with LV systolic dysfunction, and positive correlations of this marker with dilated LV and reduced LV contractility were observed. Additionally, slightly enhanced oxidative stress was shown in the remaining HCM patients with preserved LV systolic function compared to the control group. In these subgroups, oxidative stress tended to be higher in obstructive HCM compared to the nonobstructive subgroup. However, both the obstructive and nonobstructive groups were small, and the patients described by Nakamura et al. [13] were different from the typical HCM patients. The septal thickness was relatively small (especially in nonobstructive HCM with normal systolic function - mean value of $15 \mathrm{~mm}$ ), the obstructive form was defined as the LVOT gradient $>40 \mathrm{mmHg}$ (the cut-off value is usually $30 \mathrm{mmHg}$ ) and the subgroup with LV systolic dysfunction was larger than in most HCM study populations, of which they represent $5-10 \%$. In the current study, we examined more representative patients for HCM with a mean septum thickness of $24.1 \pm 3.8 \mathrm{~mm}$. Moreover, patients with LV dilation and/or systolic dysfunction were excluded.

\section{Therapeutic implication}

The current data might be of practical importance because augmented oxidative stress was abolished in an animal model of HCM [10]. Treatment with the antioxidant $\mathrm{N}$-acetylcysteine reduced myocardial oxidative stress, stress-responsive signaling kinase and fibrosis in a mouse model of HCM [10]. This antifibrotic effect was significant and was associated with a $40 \%$ reduction in the myocardial concentration of a marker of oxidative stress.

\section{Limitations}

First, the number of the patients studied is limited. Second, our analysis was based on a determination of each variable at a single time point, and with a disease with varying LVOT, the results could vary. Finally, the statistical associations reported here do not necessarily imply cause-effect relationships. Further studies are needed to validate the observations.

\section{Conclusions}

HCM patients had higher 8-iso-PGF $2 \alpha$ levels compared to the control group. Moreover, obstructive HCM is associated with the most enhanced oxidative stress in comparison to nonobstructive HCM and controls.

\section{References:}

1. Dimitrow PP, Undas A, Bober M, Tracz W, Dubiel JS: Obstructive hypertrophic cardiomyopathy is associated with enhanced thrombin generation and platelet activation. Heart, 2008, 94, e21.

2. Dimitrow PP, Undas A, Bober M, Tracz W, Dubiel JS: Plasma biomarkers of endothelial dysfunction in patients with hypertrophic cardiomyopathy. Pharmacol Rep, 2007, 59, 715-720.

3. Dimitrow PP, Krzanowski M, Nizankowski R, Szczeklik A, Dubiel JS: Comparison of the effect of verapamil and propranolol on response of coronary vasomotion to cold pressor test in symptomatic patients with hypertrophic cardiomyopathy. Cardiovasc Drugs Ther, 2000, 14, 643-650.

4. Elesber AA, Best PJ, Lennon RJ: Plasma 8-iso-prostaglandin F2alpha, a marker of oxidative stress, is increased in pa- 
tients with acute myocardial infarction. Free Radic Res, 2006, 40,385-391.

5. Forbes JM, Coughlan MT, Cooper ME: Oxidative stress as a major culprit in kidney disease in diabetes. Diabetes, 2008, 57, 1446-1454.

6. Henderson BC, Tyagi N, Ovechkin A, Kartha GK, Moshal KS, Tyagi SC: Oxidative remodeling in pressure overload induced chronic heart failure. Eur J Heart Fail, 2007, 9, 450-457.

7. Jacob MH, Pontes MR, Araújo AS, Barp J, Irigoyen MC, Llesuy SF, Ribeiro MF, Belló-Klein A: Aortic-banding induces myocardial oxidative stress and changes in concentration and activity of antioxidants in male Wistar rats. Life Sci, 2006, 79, 2187-2193.

8. Kai H, Mori T, Tokuda K, Takayama N, Tahara N, Takemiya K, Kudo $\mathrm{H}$ et al.: Pressure overload-induced transient oxidative stress mediates perivascular inflammation and cardiac fibrosis through angiotensin II. Hypertens Res, 2006, 29, 711-718.

9. Lewis JF, Maron BJ: Elderly patients with hypertrophic cardiomyopathy: a subset with distinctive left ventricular morphology and progressive clinical course late in life. J Am Coll Cardiol, 1989, 13, 36-45.

10. Marian AJ, Senthil V, Chen SN, Lombardi R: Antifibrotic effects of antioxidant $\mathrm{N}$-acetylcysteine in a mouse model of human hypertrophic cardiomyopathy mutation. J Am Coll Cardiol, 2006, 47, 827-834.

11. Minuz P, Fava C, Lechi A: Lipid peroxidation, isoprostanes and vascular damage. Pharmacol Rep, 2006, 58 Suppl., 57-68.

12. Moens AL, Takimoto E, Tocchetti CG, Chakir K, Bedja D, Cormaci G, Ketner EA et al.: Reversal of cardiac hy- pertrophy and fibrosis from pressure overload by tetrahydrobiopterin: efficacy of recoupling nitric oxide synthase as a therapeutic strategy.Circulation, 2008, 117, 2626-2636.

13. Nakamura K, Kusano KF, Matsubara H, Nakamura Y,

Miura A, Nishii N, Banba K et al.: Relationship between oxidative stress and systolic dysfunction in patients with hypertrophic cardiomyopathy. J Card Fail, 2005, 11, 117-137.

14. Sawyer DB, Siwik DA, Xiao L, Pimentel DR, Singh K, Colucci WS: Role of oxidative stress in myocardial hypertrophy and failure. J Mol Cell Cardiol, 2002, 34, 379-388.

15. Seddon M, Looi YH, Shah AM: Oxidative stress and redox signalling in cardiac hypertrophy and heart failure. Heart, 2007, 93, 903-907.

16. Takimoto E, Champion HC, Li M, Ren S, Rodriguez ER, Tavazzi B, Lazzarino G et al.: Oxidant stress from nitric oxide synthase-3 uncoupling stimulates cardiac pathologic remodeling from chronic pressure load. J Clin Invest, 2005, 115, 1221-1231.

17. Thaman R, Gimeno JR, Murphy RT, Kubo T, Sachdev B, Mogensen J, Elliott PM, McKenna WJ: Prevalence and clinical significance of systolic impairment in hypertrophic cardiomyopathy. Heart, 2005, 91, 920-925.

18. Vassalle C, Botto N, Andreassi MG, Berti S, Biagini A: Evidence of enhanced 8-isoprostane plasma levels, as index of oxidative stress in vivo, in patients with coronary artery disease. Coron Artery Dis, 2003, 14, 213-218.

Received:

October 24, 2008; in revised form: April 7, 2009. 